\title{
Crystal structure refinement and electronic properties of $\mathrm{Si}(\mathrm{cI16})$
}

\begin{tabular}{|r|l|}
\hline Journal: & Zeitschrift für Anorganische und Allgemeine Chemie \\
\hline Manuscript ID: & zaac.200900051 \\
\hline Diley - Manuscript type: & Article \\
\hline Complete List of Authors: & $\begin{array}{l}\text { Wosylus, Aron; MPI CPfS, Chemische Metallkunde } \\
\text { Rosner, Helge; MPI CPfS, Chemische Metallkunde } \\
\text { Schnelle, Walter; MPI CPfS, Chemische Metallkunde } \\
\text { Schwarz, Ulrich; MPI CPfS, Chemische Metallkunde }\end{array}$ \\
\hline Keywords: & $\begin{array}{l}\text { Silicon, Allotropy, Crystal Structure, Electronic Density of States, } \\
\text { Physical Properties }\end{array}$ \\
\hline \multicolumn{2}{|l}{} \\
\hline
\end{tabular}

\section{S ScholaroNE \\ Manuscript Central}




\title{
Crystal structure refinement and electronic properties of $\operatorname{Si}(c I 16)$
}

\author{
Aron Wosylus, Helge Rosner, Walter Schnelle, Ulrich Schwarz* \\ Max-Planck-Institut für Chemische Physik fester Stoffe, Dresden \\ Dedicated to Prof. Reinhard Nesper on the occasion of his $60^{\text {th }}$ birthday
}

\begin{abstract}
Si}(\mathrm{cl16})$ is prepared in polycrystalline form at $12(1.5) \mathrm{GPa}$ at temperatures between $800(80) \mathrm{K}$ and $1200(120) \mathrm{K}$. The crystal structure is refined by a full-profile method using x-ray powder diffraction data. $\mathrm{Si}\left(c\right.$ I16) is diamagnetic $\left(\chi_{0}=-5.6(1.8) \times 10^{-6} \mathrm{emu} \mathrm{mol}^{-1}\right)$ and shows a weakly temperature-dependent electrical resistivity $\left(\rho(300 \mathrm{~K})=0.3 \times 10^{-3} \Omega \mathrm{m}\right)$. Computations of structural and electronic properties of $\mathrm{Si}(\mathrm{cl16})$ within the local density approximation evidence the metastable character of the allotrope with respect to diamond-type silicon. The calculations yield a positional parameter which is in perfect agreement with the refined value. In agreement with the experimentally observed conductivity properties, the computed density of states evidence that the Fermi level of $\mathrm{Si}(\mathrm{cI} 16)$ is located in a pseudo-gap.
\end{abstract}

Keywords: Silicon; Allotropy; Crystal Structure; Electronic Density of States; Physical Properties

\footnotetext{
*PD Dr. U. Schwarz

Max-Planck-Institut für Chemische Physik fester Stoffe

Nöthnitzer Straße 40, 01187 Dresden, Germany

Fax: (+49) 351-4646-4002

E-mail:schwarz@cpfs.mpg.de
} 


\section{Introduction}

In the course of investigations on binary tetrels of alkaline-earth metals manufactured at high-pressure hightemperature conditions [1-4], we observed the metastable element modifications $\operatorname{Ge}(t P 12)[5,6]$ and $\operatorname{Si}(c I 16)$ [7] as minority phases after application of pressures slightly above ten GPa with a large-volume two-stage multianvil device. $\mathrm{Si}(\mathrm{cI} 16)$ was described for the first time more than forty years ago. The crystal structure (Fig. 1) was solved on the basis of $\mathrm{x}$-ray powder diffraction data, but the position parameter of silicon was only estimated by visual comparison of experimental and calculated x-ray diffraction intensities. In the atomic arrangement which is isotypic to $\mathrm{Ge}(\mathrm{cl} 16)$ [8], four-coordinated silicon atoms are reported to adopt a trigonal pyramidal coordination with $d(\mathrm{Si}-\mathrm{Si})$ of $1 \times 2.369(9) \AA$ and $3 \times 2.384(9) \AA[7]$ instead of tetrahedral with $d(\mathrm{Si}-\mathrm{Si})=2.351$ $\AA$ [9] in ambient pressure $\mathrm{Si}(c F 8)$.

Later in-situ investigations at high pressures [10] including studies of the formation of $\mathrm{Si}(c 116)$ at elevated temperatures $[11,4]$ as well as determinations of the electrical resistance were performed on minute samples using the diamond anvil cell technique [12]. The results evidence that, at ambient temperature, the phase $\operatorname{Si}(c I 16)$ is metastable between ambient pressure and at least $2 \mathrm{GPa}$. Transformation at normal pressure requires heating to $423 \mathrm{~K}$ for several hours [7].

The renewed interest in crystal structure and physical properties of $\operatorname{Si}(c I 16)$ is motivated by the lack of magnetic measurements and refined structural data as well as by the importance of the allotrope as a prototype tetrahedral framework with a differentiation of interatomic distances due to a decrease of symmetry. We report here the least squares refinement of crystal structure parameters using x-ray powder diffraction data and measurements of magnetic properties and electrical transport features. Concomitant band structure calculations independently evaluate the stability of the allotrope and the refined structural parameter. Moreover, they shed light on the electronic structure and the specific changes caused by the deviation of the coordination polyhedron from tetrahedral symmetry.

\section{Experiments and band structure calculations}

$\mathrm{Si}(c 116)$ was synthesized from the starting material $\mathrm{Si}(c F 8)$ (Alpha Aesar 99.9999 \%) at 12(1.5) GPa and temperatures between $800(100) \mathrm{K}$ and $1200(120) \mathrm{K}$ in a hydraulic press equipped with a Walker-type module $[13,14]$. Typically, pressure was increased within $4 \mathrm{~h}$ and released within $10 \mathrm{~h}$. Pressure calibration had been performed prior to the experiments measuring the resistance changes associated with the phase transitions of $\mathrm{Bi}$ [15] and $\mathrm{Pb}$ [16-18]. The temperature-current relation had been determined in separate runs with $\mathrm{W} / \mathrm{WRe}$ thermocouples. Heating was realized with graphite tubes. Hexagonal boron nitride served as crucible material. No apparent reaction with silicon at the selected transformation conditions was observed. X-ray powder diffraction experiments were performed in transmission arrangements (Huber Image Plate $\mathrm{G} 670$ with $\mathrm{Cu}_{\mathrm{Ka}}$ radiation and at ID31 of the ESRF using a high-resolution set-up and synchrotron radiation). Details concerning measurement and refinement as well as selected results are given in Tab. 1.

The samples for the physical measurements on $\mathrm{Si}(\mathrm{cI} 16)$ were two polycrystalline pieces from separate highpressure synthesis runs. The magnetization was determined in magnetic fields $\mu_{0} H=3.5 \mathrm{~T}$ and $7 \mathrm{~T}$ between 1.8 $\mathrm{K}$ and $300 \mathrm{~K}$ and also at lower fields in a SQUID magnetometer (MPMS XL-7, Quantum Design). The sample 
consisted of 2 pieces with a total mass of $27.82 \mathrm{mg}$ co-mounted with GE-7031 varnish to a thin quartz rod. Corrections for the sample holder and the glue were applied. The electrical resistance was measured by a linear four-point method using alternating current in the temperature range $1.8-380 \mathrm{~K}$. For that purpose one piece was ground to dimensions $1.85 \times 1.90 \times 1.14 \mathrm{~mm}^{3}$. Due to the uncertainties of the contact distance and the crosssection the estimated inaccuracy of the electrical resistivity is $\pm 30 \%$. For comparison the resistivity data of another piece (dc measurement, $4-320 \mathrm{~K}$ ) are given.

For electronic structure calculations the full-potential local-orbital scheme FPLO (version: FPLO 8.00-31) within the local density approximation (LDA) was used [19]. In the scalar relativistic calculations the exchange and correlation potential of Perdew and Wang was chosen [20]. To ensure accurate total energy and density of states (DOS) information, a fine, well converged $k$-mesh of 9486 points in the irreducible part of the Brillouin zone was used.

\section{Results and Discussion}

Within the investigated pressure and temperature range, syntheses yields polycrystalline single-phase $\mathrm{Si}(c I 16)$ in all experiments. X-ray powder diffraction patterns (Fig. 2) reveal a significant peak broadening, e.g., for the strongest reflection (121) a full width at half maximum of $0.08^{\circ}$ at $2 \theta=8.477^{\circ}$. By applying the Scherrerequation [21] this corresponds to an average domain size of roughly $250 \AA$. The small particle dimension is attributed to a first-order transition of the intermediate high-pressure phase $\mathrm{Si}(h R 8)$ into $\mathrm{Si}(c 116)$ at pressures around $2 \mathrm{GPa}[10]$ upon decompression.

The structure model of the earlier solution is refined by means of a least squares procedure using complete $\mathrm{x}$-ray powder diffraction diagrams (Fig. 2). The supposed atomic arrangement [7] is essentially confirmed by the results with the main exception that the present analysis of the high-quality synchrotron x-ray diffraction intensities evidences a difference of the crystallographically independent interatomic distances of about $0.06 \AA$ (see Tab. 1). Thus, $\mathrm{Si}($ cI16) belongs indeed to the small, but still growing set of high-pressure phases in which the reduction of point symmetry is associated with a differentiation of chemically equivalent interatomic distances [22].

The high-field magnetic susceptibility $\chi(T)=M / H$ (Fig. 3, top) of the sample is slightly dependent on field, which indicates minor ferromagnetic impurities. The latter are taken into account by the Honda-Owen extrapolation for each temperature. The weakly temperature-dependent correction applied to the $7 \mathrm{~T}$ data corresponds to approximately $-1.5 \times 10^{-6} \mathrm{emu} \mathrm{mol}^{-1}$, which would be consistent with, e.g., a mass content of elemental $\mathrm{Fe}$ of $16 \times 10^{-6}$. The resulting susceptibility still displays a weak Curie contribution. The intrinsic, essentially temperature-independent susceptibility of $\mathrm{Si}(c I 16)$ is obtained by a fit: $\chi_{0}(T=0 \mathrm{~K})=-5.6(1.8) \cdot 10^{-6}$ $\mathrm{emu} \mathrm{mol}^{-1}$, the value of $\chi(300 \mathrm{~K})$ is $-5.3(1.5) \cdot 10^{-6} \mathrm{emu} \mathrm{mol}^{-1}$. For comparison, the value of $\chi(300 \mathrm{~K})$ for $\mathrm{Si}(c F 8)$ is $-3.12 \cdot 10^{-6} \mathrm{emu} \mathrm{mol}^{-1}$ [23] or, according to a different source, $-5.3 \cdot 10^{-6} \mathrm{emu} \mathrm{mol}^{-1}$ [24]. No phase transitions are observed in the covered temperature range.

The temperature dependence of the electrical resistivity (Fig. 3, bottom) is rather weak: from a room-temperature value $\rho(300 \mathrm{~K})=0.3 \cdot 10^{-3} \Omega \mathrm{m}$ the resistivity increases only to $\rho_{0}=1.0 \cdot 10^{-3} \Omega \mathrm{m}$ at $1.8 \mathrm{~K}$. The value at $100 \mathrm{~K}$ of $0.6 \cdot 10^{-3} \Omega \mathrm{m}$ compares very well with that in a previous investigation $\left(\rho(100 \mathrm{~K})=0.5 \cdot 10^{-3} \Omega \mathrm{m}[12]\right)$. In order to check the variation among different samples and primings, we measured a second piece from another synthesis run (see Fig. 3b). The resistivity is slightly higher but shows almost the same temperature dependence. 
For $T>50 \mathrm{~K}$ and at least up to $350 \mathrm{~K}$ the conductivity $\sigma(T)$ of our $\operatorname{Si}(c I 16)$ samples increases linearly with temperature. The same observation for $\sigma(T)$ has been previously attributed to localization effects in a disordered system [12]. Below $10 \mathrm{~K}$, the equation $\sigma(T)=\sigma_{0}+a T^{\alpha}$ with $\alpha=3 / 2$ can be well fitted to the recent conductivity measurements while in an analysis of earlier data a power law with $\alpha=2$ has been applied [12].

As an independent approach to both the electronic and the structural features of metastable $\mathrm{Si}(c 116)$, we applied a highly accurate full-potential band structure scheme to calculate electronic structure and total energies for $\mathrm{Si}(c 116)$. As a reference, well-known diamond-type $\mathrm{Si}(c F 8)$ is selected. For the experimentally determined lattice parameters at ambient conditions, diamond-type silicon is more stable than the metastable cubic allotrope by an energy difference of about $130 \mathrm{meV}$ per atom $(12.6 \mathrm{~kJ} / \mathrm{mol})$. For the same unit cell volume, the positional parameter $x$ for $\operatorname{Si}(c I 16)$ was optimized (Fig. 4). The equilibrium position is calculated to $x_{\text {theo }}=0.10149(5)$ in excellent agreement with the experimental value $x_{\exp }=0.10143(4)$ (see Tab. 1 and comment therein).

Figure 5 shows a comparison of the calculated electronic $\mathrm{DOS}$ of $\mathrm{Si}(c 116)$ and $\mathrm{Si}(c F 8)$. We find semiconducting behaviour for the diamond-type phase of silicon with a DOS gap of about $0.5 \mathrm{eV}$. The underestimation with respect to the experimental value of about $1.1 \mathrm{eV}$ is related to the well-known gap problem of the LDA. In contrast, for $\mathrm{Si}(\mathrm{cI16})$ we find that the Fermi level $\varepsilon_{\mathrm{F}}$ is located in a pronounced pseudo-gap with a low density of states. A corresponding calculation for a model with equidistant neighbours provides rather large values for the DOS at $\varepsilon_{\mathrm{F}}$. Thus, the emergence of the local DOS minimum for $\mathrm{Si}(c I 16)$ is clearly caused by the differentation of the distances $d(\mathrm{Si}-\mathrm{Si})$ within the trigonal pyramidal coordination.

The valence band width of $\mathrm{Si}(c I 16)$ is slightly larger (about $1 \mathrm{eV}$ ) than that of $\mathrm{Si}(c F 8)$. A model with four equivalent distances $d(\mathrm{Si}-\mathrm{Si})$ and an additional adjustment of the interatomic distances to the value of diamondtype silicon yields a valence band width which is very close to that of $\operatorname{Si}(c F 8)$. This finding reveals that the slightly larger valence band width of $\mathrm{Si}(c I 16)$ compared to diamond-type silicon is mainly caused by the shorter next-neighbour distance.

As a summary, we have collected additional theoretical and experimental evidence for the difference of the symmetry-independent interatomic distances in the trigonal pyramidal coordination sphere of silicon atoms in $\mathrm{Si}($ cl16). In accordance with the findings of resistivity measurements, band structure calculations reveal substantial differences of the electronic features which can be traced back to the dissimilarity of the atomic arrangements of $\mathrm{Si}(c F 8)$ and $\mathrm{Si}(c I 16)$. The results concerning total energy and atomic position establish elaborate band structure calculations once more as a valuable and reliable tool for gaining deeper insight into crystal structure properties and phase diagrams of elements $[25,26]$.

\section{Acknowledgement}

For supporting high-pressure synthesis we thank Carola J. Müller, Susann Leipe and Knut Range. Help with the high-resolution x-ray powder diffraction measurements by Irene Margiolaki at the ESRF is gratefully acknowledged. Special thanks to Ralf Koban for fastidious sample priming. 


\section{References}

[1] A. Wosylus, Yu. Prots, U. Burkhardt, W. Schnelle, U. Schwarz, Yu. Grin, Solid State Sci. 2006, 8, 773.

[2] A. Wosylus, Yu. Prots, U. Burkhardt, W. Schnelle, U. Schwarz, Yu. Grin, Z. Naturforschung, B 2006, 61, 1485.

[3] A. Wosylus, Yu. Prots, U. Burkhardt, W. Schnelle, U. Schwarz, Sci. Technol. Adv. Mater. 2007, 8, 383.

[4] A. Wosylus, PhD Thesis, University of Dresden, submitted.

[5] F. P. Bundy, J. S. Kasper, Science 1963, 139, 340.

[6] A. Wosylus, Yu. Prots, W. Schnelle, U. Schwarz, Z. Naturforschung, B 2008, 63, 608.

[7] R.H. Wentorf Jr., J.S. Kasper, Science 1963, 139, 338.

[8] R. J. Nelmes, M. I. McMahon, N. G. Wright, D. R. Allan, J. S. Loveday, Phys. Rev. B 1993, 48, 9883.

[9] R.R. Reeber and B.A. Kulp, Trans. Met. Soc. AIME 1965, 233, 698.

[10] J. Crain, G.J. Ackerland, J.R. Maclean, R.O. Piltz, P.D. Hatton, G.S. Pawley, Phys. Rev. B 1994, 50, 13043.

[11] G. Weill, J. L. Mansot, G. Sagon, C. Carlone, J. M. Besson, Semicond. Sci. Technol. 1989, 4, 280.

[12] J.M. Besson, E.H. Mokhtari, J. Gonzalez, G. Weill, Phys. Rev. Lett. 1987, 59, 473.

[13] D. Walker, M.A. Carpenter, C.M. Hitch, Am. Mineral. 1990, 75, 1020.

[14] H. Huppertz, Z. Kristallogr. 2004, 219, 330.

[15] I. C. Getting, Metrologia 1998, 35, 119.

[16] T. Takahashi, H. Kwang, W.A. Bassett, Science 1969, 165, 1352.

[17] L.F. Vereshchagin, A.A. Semerchan, N.N. Kuzin, Y.A. Sadkov, Sov. Phys. Dokl. 1970, 15, 295.

[18] H. Mii, I. Fujishiro, M. Senoo, K. Okawa, High Temp.-High Pressures 1973, 5, 155.

[19] K. Koepernik, and H. Eschrig, Phys. Rev. B 1999, 59, 1743.

[20] J.P. Perdew and Y. Wang, Phys. Rev. B 1992, 45, 13244.

[21] P. Scherrer, Nachr. Ges. Wiss. Göttingen 1918, 100.

[22] R. Demchyna, S. Leoni, H. Rosner, U. Schwarz, Z. Kristallogr. 2006, 221, 420.

[23] CRC Handbook of Chemistry and Physics, 87 $7^{\text {th }}$ ed., D. R. Lide (Ed.), CRC Press, Boca-Raton 2007.

[24] P. W. Selwood, Magnetochemistry, $2^{\text {nd }}$ ed., Interscience, New York 1956.

[25] U. Schwarz, L. Akselrud, H. Rosner, A. Ormeci, Yu. Grin, M. Hanfland, Phys. Rev. B 2003, 67, 214101.

[26] A. Ormeci, K. Koepernik, and H. Rosner, Phys. Rev. B 2006, 74, 104119.

[27] L.G. Akselrud, P.Yu. Zavalii, Yu. Grin, V.K. Pecharski, B. Baumgartner, E. Wölfel, Mater. Sci. Forum 1993, 133.

[28] J. Rodriguez-Carvajal, Physica B 1993, 192, 55. 


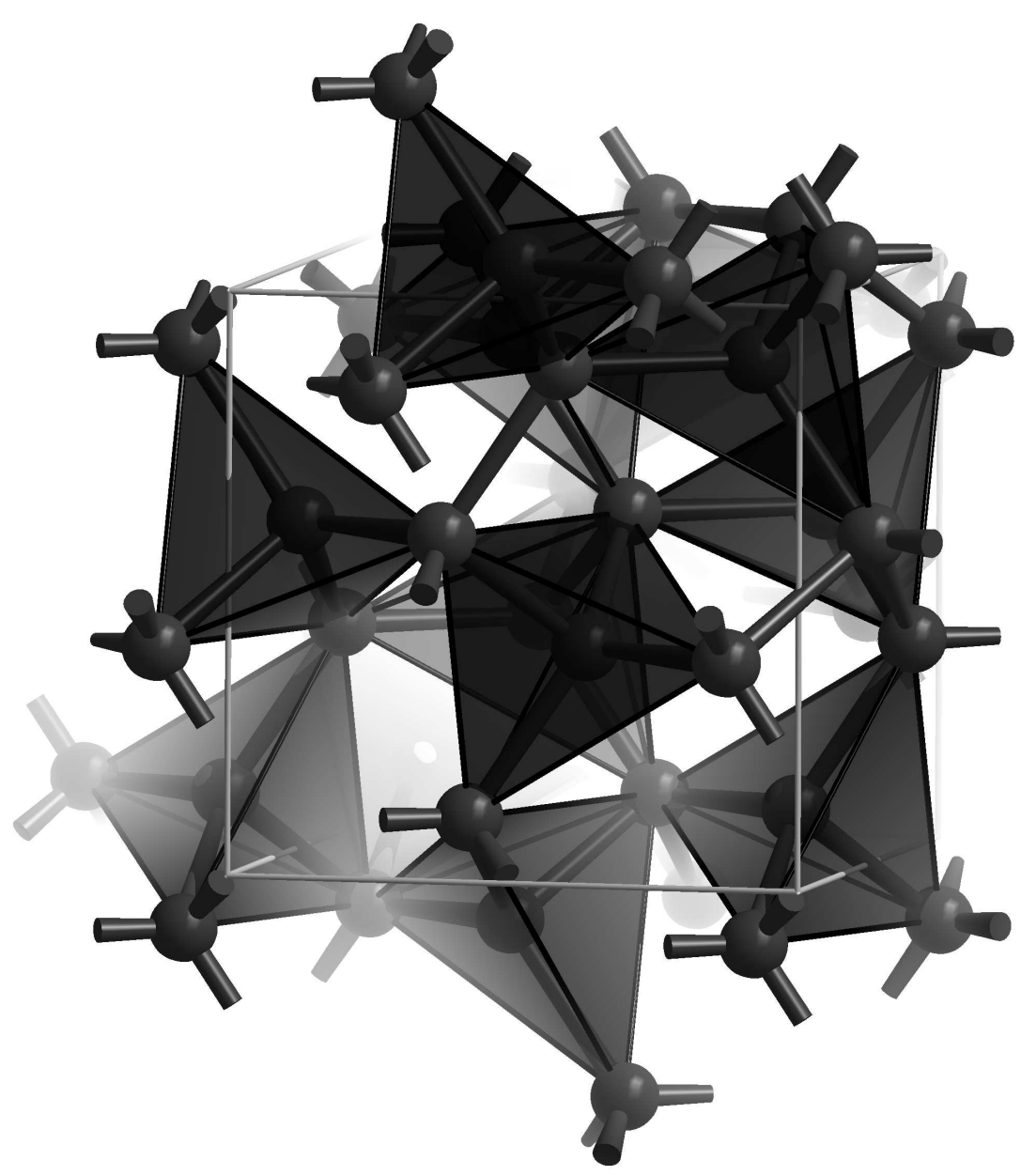

Fig. 1. Crystal structure of $\mathrm{Si}(\mathrm{cI} 16)$. Short interatomic distances $d(\mathrm{Si}-\mathrm{Si})=2.35 \pm 0.04 \AA$ which are compatible with covalent single bonds are emphasized by dark lines. Distorted trigonal pyramidal coordination polyhedra are shaded. The cubic unit cell is indicated by thin grey lines. $624 \times 624 \mathrm{~mm}(96 \times 96 \mathrm{DPI})$ 


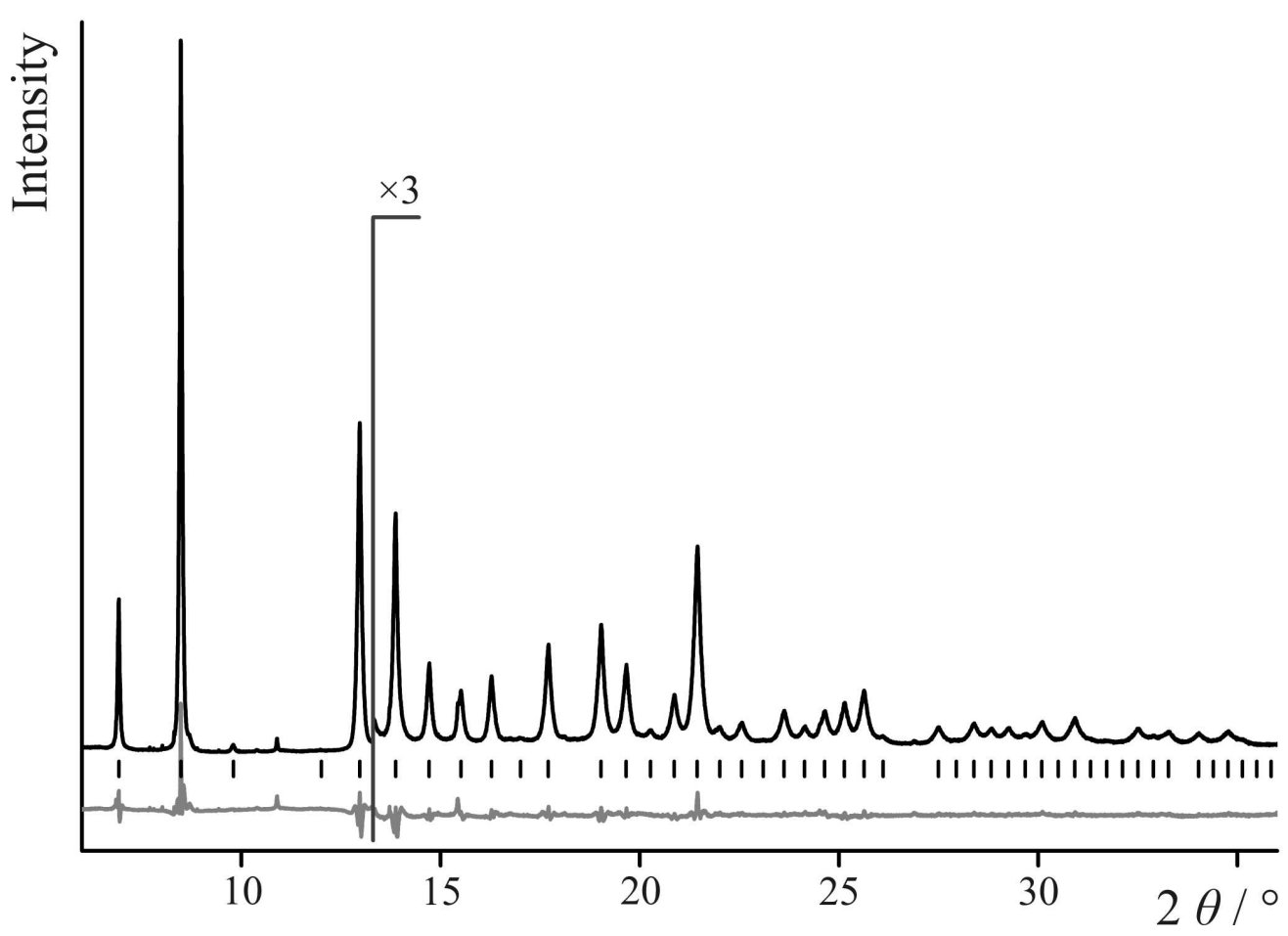

Fig. 2. X-ray powder diffraction diagram of $\mathrm{Si}(\mathrm{CI} 16)$ recorded using low-divergence synchrotron radiation at ID31 of the $\operatorname{ESRF}(\lambda=0.39987(2) \AA)$. The grey curve corresponds to the difference between observed and calculated intensities multiplied by a factor of three. Reflection positions are indicated by vertical bars.

$87 \times 62 \mathrm{~mm}(600 \times 600 \mathrm{DPI})$ 


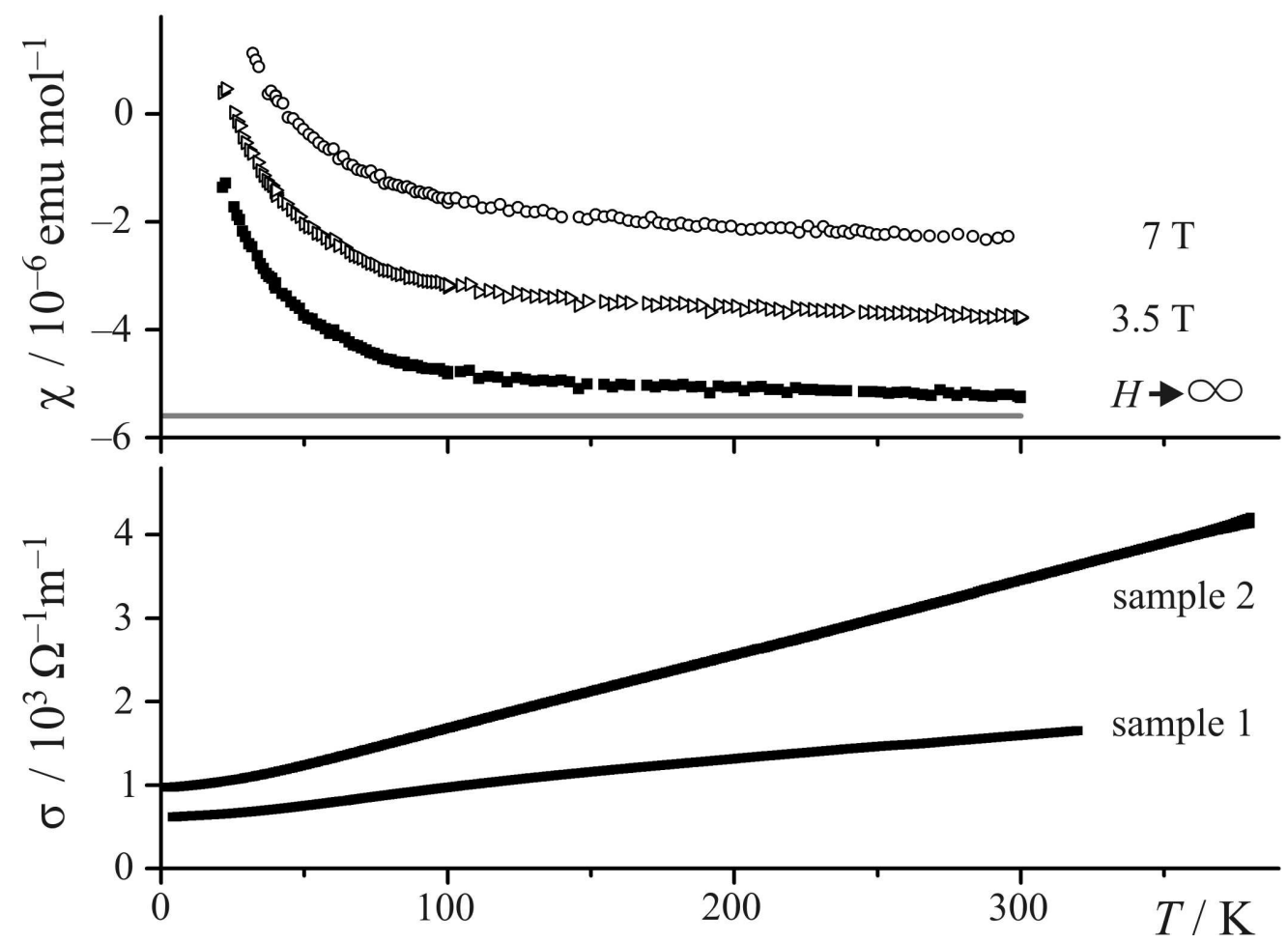

Fig. 3. Top: Measured $(\mu \mathrm{OH}=7.0 \mathrm{~T}$ and $3.5 \mathrm{~T})$ and extrapolated $(\mu \mathrm{OH} \rightarrow \boldsymbol{\infty})$ molar magnetic susceptibility $\chi(T, H)$ of the Si(CI16) sample. The grey line indicates the intrinsic temperatureindependent term $\chi_{0}$ for $T=0 \mathrm{~K}$. Bottom: Electrical conductivity $\sigma(T)$ of two Si(cI16) samples. The linear dependence above approximately $50 \mathrm{~K}$ is consistent with a heavily doped semiconductor. $88 \times 65 \mathrm{~mm}(600 \times 600 \mathrm{DPI})$ 


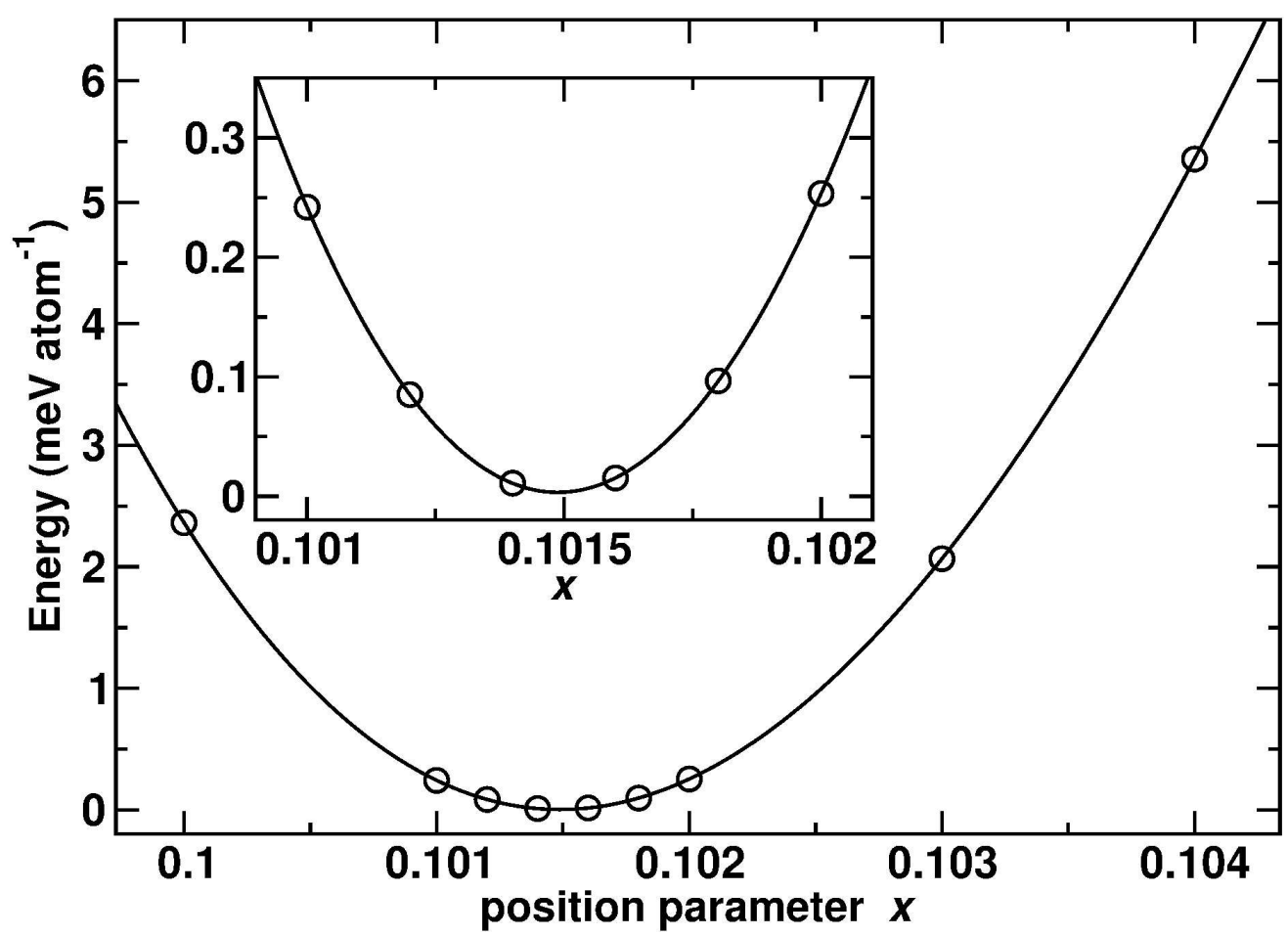

Fig. 4. Total energy as a function of the silicon coordinate. Open circles indicate calculated values, black lines represent a least squares fit of a fourth-order polynomial to the data. The minimum corresponds to $\mathrm{x}=0.10149(5)$ in perfect agreement with the experimental result. The insert shows an enlargement of the curve in the vicinity of the energy minimum. $236 \times 170 \mathrm{~mm}(600 \times 600 \mathrm{DPI})$ 


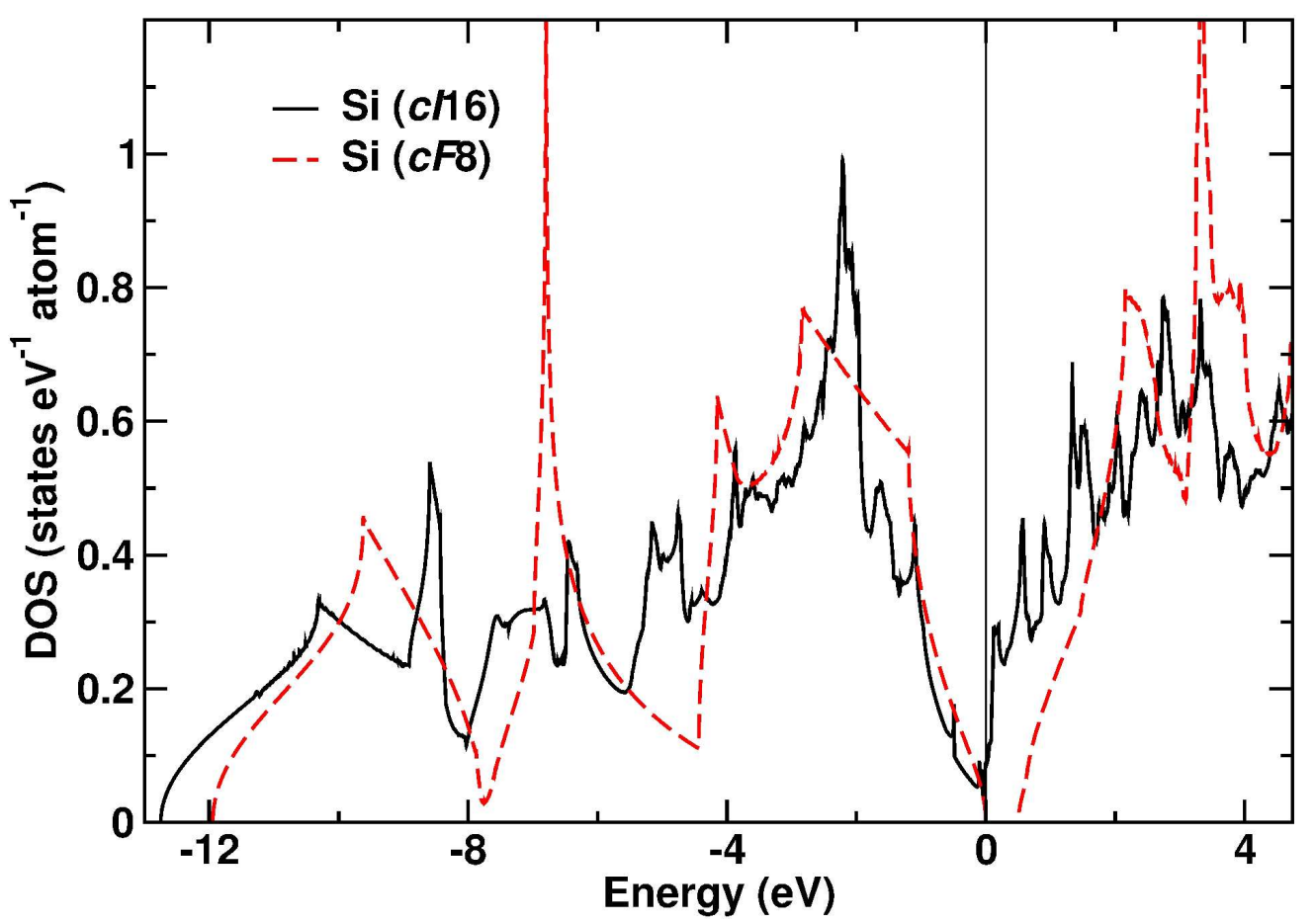

Fig. 5. Electronic density of states of $\mathrm{Si}(\mathrm{cF} 8)$ and $\mathrm{Si}(\mathrm{CI} 16)$. The LDA calculations reveal semiconducting behaviour for diamond-type silicon and a pseudo-gap with a low density of states for the metastable phase. $242 \times 170 \mathrm{~mm}(600 \times 600 \mathrm{DPI})$ 
1

2

3

4

5

6

7

8

9

Tab. 1. Details of the diffraction measurements and crystallographic data of $\mathrm{Si}($ cl16). Refinements are performed with the computer program WinCSD [27] Rietveld refinements are performed using Fullprof [28]. A Pseudo-Voigt function is used for profile simulation and a sixth order polynomial for modelling the background.

\begin{tabular}{ll}
\hline Compound & $\operatorname{Si}(c I 16)$ \\
Space group (No.) & $I a-3(206)$ \\
Lattice parameters: & \\
Rietveld refinement of synchrotron data; & $6.6265(1) \AA ;$ \\
Laboratory source with $\mathrm{LaB}_{6}$ standard; & $6.6229(2) \AA ;$ \\
Earlier data & $6.64(1)[7]$ \\
Unit cell volume / $\AA^{3}$ & $290.97(1)$ \\
Refined position parameter $\operatorname{Si}(x, x, x)^{*} ;$ & $0.10143(4) ;$ \\
Earlier data & $0.103(1)[7] ; 0.1025[10]$ \\
$U_{11}=U_{22}=U_{33}=U_{\text {iso }} / \AA^{2}$, & $0.0058(2)$, \\
$U_{12}=U_{13}=U_{23} / \AA^{2}$ & $0.0003(2)$ \\
$Z$ & 16 \\
Calculated density / g cm ${ }^{-3} ;$ Earlier data & $2.564 ; 2.55[7]$ \\
Diffractometer, & ESRF ID31, \\
wave length $\lambda / \AA$ & $0.39987(2)$ \\
Diffraction set-up, & Transmission alignment, \\
Quartz capillary & $d=1$ mm \\
Number of reflections, & 97, \\
$2 \theta_{\text {max }} /$ Degree, step width / degree & $36,0.005$ \\
Residuals $R_{\mathrm{p}} ; R_{\text {wp }} ; R_{\text {exp }}$ & $5.82 ; 7.84 ; 1.65$ \\
Interatomic distances with experimental & $1 \times 2.3283(4) \AA ; 2.369(9) \AA[7]$ \\
errors as calculated in the full profile & $3 \times 2.3841(4) \AA ; 2.384(9) \AA[7]$ \\
refinements *; Earlier data & $1 \times 3.4104(4) \AA ; 3.381(9) \AA[7]$ \\
Angles / Degree; Earlier data & $98.70(1) ; 98.1(3)[7]$ \\
& $117.75(1) ; 118.0(4)[7]$ \\
\hline
\end{tabular}

* The maximal error of the internal coordinate as estimated on the basis of the number of observed reflections corresponds to 0.002 . 